\title{
30707 T-Stent or Half T-Stent-Assisted Technical Embolization of Wide-Necked Aneurysms at the Internal Carotid- Posterior Communicating Artery Bifurcation
}

Takato Nakajo, ${ }^{1,2}$ Tomoaki Terada, ${ }^{1}$ Hideo Okada, ${ }^{3}$ Hiroaki Sakaguchi, ${ }^{1}$ Syun Takano, ${ }^{1}$ Akira Nishiyama, ${ }^{1}$ Arisa Umezaki, ${ }^{1}$ Yuko Tanaka, ${ }^{1}$ Hiroo Yamaga, ${ }^{1}$ Hiroaki Matsumoto, ${ }^{1}$ and Tohru Mizutani ${ }^{2}$

Objective: We report the usefulness and pitfalls of coil embolization using the T or half T-stent technique for aneurysms located at internal carotid artery-posterior communicating artery (ICA-P-com) bifurcation in which the neck is wide and the P-com must be kept patent due to it being the fetal-type with a hypoplastic P1 segment.

Case Presentations: Two cases were treated using the T-stent technique and two were treated using the half T-stent technique. The average age of the patients was 70.3 years and all were females. One aneurysm ruptured. The average size of aneurysms and neck was $12 \mathrm{~mm}$ and $8.5 \mathrm{~mm}$, respectively, in the T-stent group, and $7.4 \mathrm{~mm}$ and $6.7 \mathrm{~mm}$, respectively, in the half T-stent group. An S- or pigtail-shaped microcatheter (MC) was used to navigate into the P-com. Stent deployment was successful in all the cases. Retreatment was required in one case treated using the T-stent technique due to major recurrence. Conclusion: T or half T-stent-assisted coil embolization can be an alternative endovascular treatment method for widenecked ICA-P-com aneurysms in which the P-com must be kept patent due to it being the fetal-type with a hypoplastic $\mathrm{P} 1$ segment.

Keywords coil embolization, T-stent technique, half T-stent technique, internal carotid posterior communicating artery bifurcation, wide-necked aneurysms

\section{Introduction}

Endovascular treatment of wide-necked aneurysms at the internal carotid artery-posterior communicating artery (ICAP-com) bifurcation is difficult if a fetal-type P-com arises

\footnotetext{
${ }^{1}$ Department of Neurosurgery, Fujigaoka Hospital, Showa University, Yokohama, Kanagawa, Japan

${ }^{2}$ Department of Neurosurgery, Showa University School of Medicine, Tokyo, Japan

${ }^{3}$ Wakayama Rosai Hospital, Wakayama, Wakayama, Japan
}

Received: June 10, 2020; Accepted: December 20, 2020

Corresponding author: Takato Nakajo. Department of Neurosurgery, Showa University School of Medicine, 1-5-8 Hatanodai, Shinagawa-ku, Tokyo 142-8666, Japan

Email: takatosp@med.showa-u.ac.jp

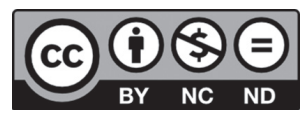

This work is licensed under a Creative Commons Attribution-NonCommercialNoDerivatives International License.

(C)2021 The Japanese Society for Neuroendovascular Therapy from the dome and is accompanied by a hypoplastic P1 segment because the P-com must be kept patent. Recently, embolization has been performed using different stentassisted techniques for similar wide-necked aneurysms. ${ }^{1-4)}$ In addition to the T-stent technique that we employ, ${ }^{5-7)}$ there are reports of treatment using the Y-configuration ${ }^{8-10)}$ or other stenting techniques. ${ }^{11,12)}$

T-stenting is a technique for neck formation by stenting the parent artery and a branching artery without overlapping of the stents to treat bifurcation-type wide-necked aneurysms (Fig. 1A and 1B). On the other hand, half T-stenting is a technique used when the neck of the aneurysm can be covered with a single stent placed via the branching artery. We call this method the half T-stent technique because it involves part of the T-stent technique (Fig. 1C and 1D).

We report four cases of ICA-P-com aneurysms that were difficult to treat for the above reasons, and treated them using the T-stent or half T-stent technique. 


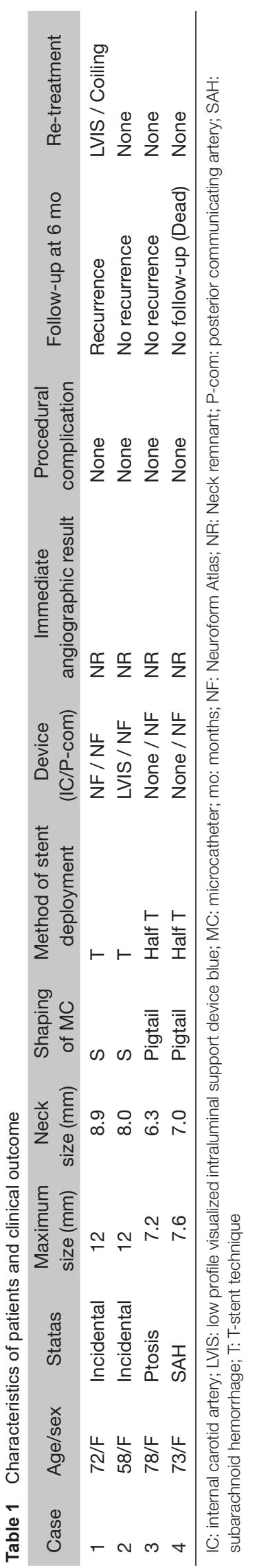

\section{Case Presentation}

Patients

Four patients with ICA-P-com aneurysms were treated by embolization using the T-stent or half T-stent technique at our hospital or related hospitals.

\section{Results (Table 1)}

The T-stent and half T-stent techniques were applied to two patients each. The patients were all females with a mean age of 70.3 (58-78) years. The two aneurysms treated by T-stenting were detected by chance. Of the two patients treated by half T-stenting, one presented with oculomotor nerve palsy and the other had a ruptured aneurysm. The mean aneurysm and neck sizes of the lesions treated by T-stenting were $12(12,12) \mathrm{mm}$ and $8.5(8.9,8.0) \mathrm{mm}$, and those of the lesions treated by half T-stenting were $7.4(7.2,7.6) \mathrm{mm}$ and $6.7(6.3,7.0) \mathrm{mm}$, respectively. To secure the P-com, the catheter was shaped into an S or pigtail shape. All stents were the Neuroform Atlas (NF) (Stryker, Kalamazoo, MI, USA). For T-stenting, NF and low profile visualized intraluminal support device (LVIS) blue (Microvention TERUMO, Tustin, CA, USA) were placed in the ICA in one patient each. Securing of the $\mathrm{P}$-com and stenting were successful in all patients, and the patency of the P-com was maintained. In addition, no perioperative complications, including those accompanied by exacerbation of neurological findings, were noted. The mean follow-up period was $6.3(1-12)$ months. The outcome immediately after embolization was neck remnant (NR), but one patient treated by T-stenting with placement of the NF in the ICA developed recurrence and was retreated.

\section{Representative case: T-stent technique}

Case 1: The patient was a 72-year-old woman in whom an unruptured aneurysm with a maximum diameter of $12 \mathrm{~mm}$ was detected by chance. The lesion increased in size and was suspected to adhere to the anterior choroidal artery (Fig. 2A-2C).

Treatment: Dual antiplatelet therapy (DAPT) was initiated 2 weeks before embolization. A 6-Fr. intermediate catheter was inserted to the IC petrous portion via an 8-Fr. guiding catheter placed in the IC. An Excelsior SL-10 (Stryker) was inserted through the aneurysm to the distal part of the middle cerebral artery (MCA) and an S-shaped Headway 17 (Microvention TERUMO) was inserted into the P-com (Fig. 2D). An Excelsior SL-10 was inserted into the 

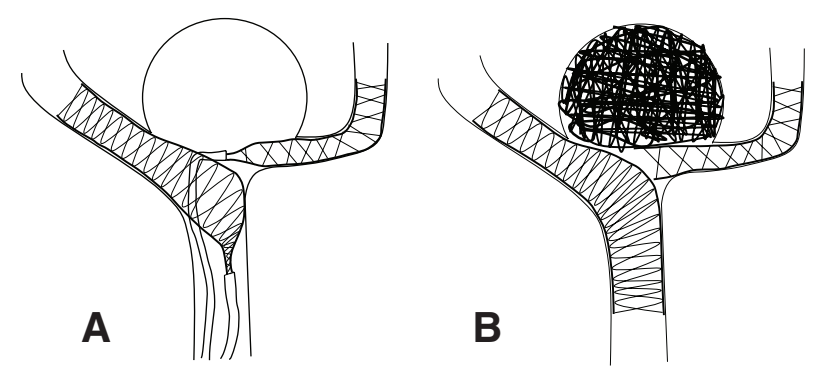

Fig. 1 T-stent technique and half T-stent technique for wide-necked aneurysms of the bifurcation. (A) Schema showing the partial deployment of two stents in the parent artery and other branch. (B) Schema showing full deployment of the stents without overlapping after partial

aneurysm via another 5-Fr. guiding catheter placed in the proximal part of the IC. An NF $4.5 \times 30 \mathrm{~mm}$ was deployed in the ICA via the MCA, and fixed without deploying the stump of the stent and with the catheter retracted to prevent the stent from sliding into the aneurysm during surgery. An NF $3.5 \times 21 \mathrm{~mm}$ was inserted to the P-com and deployed to the neck, fixed similarly without deploying the stump, and intraaneurysmal embolization was performed after assessing the state of stent deployment by cone-beam CT (Fig. 2E). Then, the stent on the ICA side and that on the P-com side were deployed, and surgery was ended in a state of NR (Fig. 2F). The patient was discharged without complication, but coil compaction was noted 6 months after surgery (Fig. 2G) and re-embolization was performed using an additional LVIS blue after 1 year due to exacerbation. No recurrence was noted for 1 year thereafter (Fig. 2H).

In Case 2, intraaneurysmal embolization was performed using a similar procedure by placing an LVIS blue $4.5 \times 23 \mathrm{~mm}$ in the ICA via the MCA and an NF $3.5 \times 21 \mathrm{~mm}$ in the P-com. There has been no recurrence for 6 months after surgery.

\section{Representative case: Half T-stent technique}

Case 3: A 78-year-old woman with an unruptured aneurysm with a maximum diameter of $7.2 \mathrm{~mm}$ presenting with blepharoptosis (Fig. 3A and 3B).

Treatment: DAPT was initiated 2 weeks before embolization. A 6-Fr. intermediate catheter was inserted to the ICA petrous portion via an 8 -Fr. guiding catheter placed in the ICA. A Headway 17 in a pigtail shape was inserted to the P-com (Fig. 3C) and an Excelsior SL-10 was inserted into the aneurysm. In the P-com, an NF $4.0 \times 21 \mathrm{~mm}$ was deployed to the neck and fixed without deploying the stump, and intraaneurysmal embolization was performed after assessing the state of stent deployment by cone-beam CT

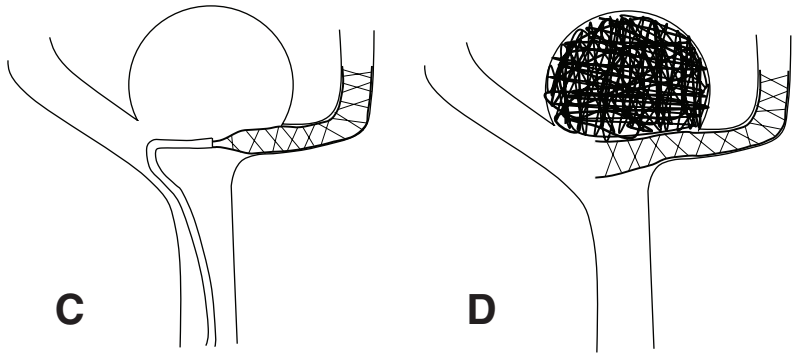

or full coil embolization. (C) Schema showing partial deployment of the stent through the non-main branch of the bifurcation. (D) Schema showing full deployment of the stent without bridging of the parent artery after partial or full coil embolization.

(Fig. 3D and 3E). The stent was deployed and surgery was ended in a state of NR (Fig. 3F). The patient was discharged without complication and no recurrence has been noted for 6 months after surgery, but blepharoptosis was not improved.

In Case 4, as craniotomy was rejected, embolization was performed by the double catheter technique on the day of onset, but patency of the P-com was unable to be maintained. Therefore, with sufficient informed consent from the family, stent-assisted embolization following DAPT loading was performed on the same day. This treatment was approved by the Advanced Novel Medical Technology/Unapproved Novel Medical Devices Evaluation Committee and ethical review board of our hospital (approval No.: 01-22, F2019C88). Although postoperative re-rupture was not observed, the patient eventually died due to severe subarachnoid hemorrhage 26 days after surgery.

None of the patients developed thrombotic or hemorrhagic complications. Although the follow-up period was not long, there has been only one case of recurrence at present and no subsequent recurrence was noted after retreatment.

\section{Discussion}

T-stent-assisted embolization was reported to be effective for bifurcation-type wide-necked aneurysms. ${ }^{5-7)}$ At our hospital, indications for endovascular treatment for cerebral aneurysms using T-stenting or half T-stenting are limited to wide-necked lesions that have inoccludable branching vessels arising from the aneurysm and are difficult to treat by direct surgery or other techniques. Furthermore, T-stenting is considered if the neck cannot be covered sufficiently by half T-stenting. The sites to which this technique can be applied include the ICA-P-com, basilar tip, basilar artery-superior cerebellar artery, and anterior communicating artery. 

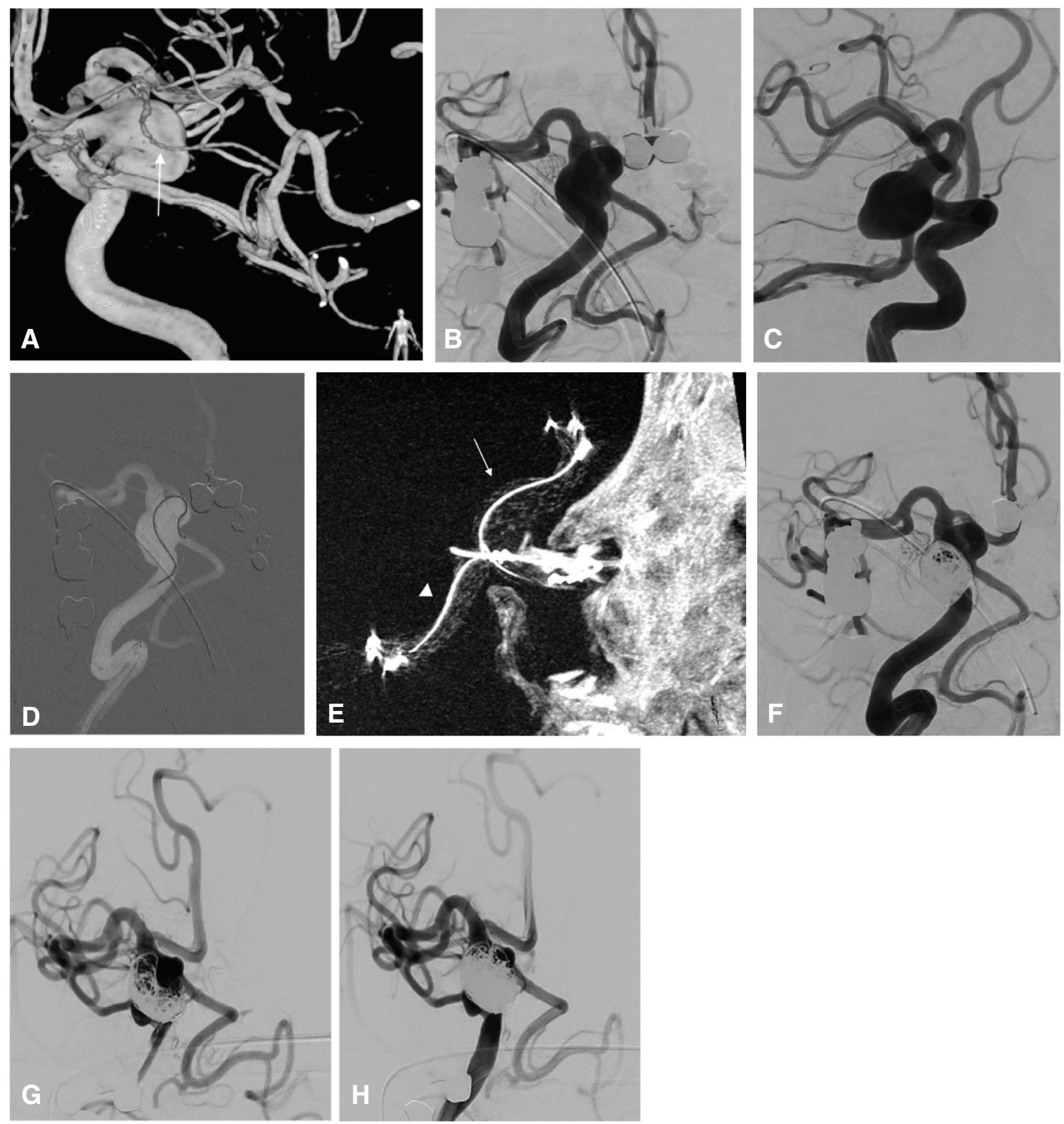

Fig. 2 (A) Reconstructed 3D-RA of the right ICA. The unruptured aneurysm was located at the right ICA-P-com. Adhesion of the anterior choroidal artery to the aneurysmal wall was predicted (arrow), (B, C) DSA of the right ICA (working angle). (D) Image before and after $\mathrm{S}$-shaped MC deployment. (E) Cone-beam CT after stent deployment (arrow: ICA, Neuroform Atlas $4.5 \times 30$, arrowhead: P-com, Neuroform

Atlas $3.5 \times 21)$. (F) DSA just after embolization. The aneurysm was obliterated with a small NR. (G) DSA 6 months after the initial embolization demonstrated recurrence of the aneurysm. (H) DSA just after retreatment (additional stent deployment and coil embolization). 3D-RA: 3D-rotational angiography; ICA-P-com: internal carotid artery-posterior communicating artery; NR: neck remnant

Half T-stenting is used to reduce the neck size and is not expected to provide flow-diverting effects. Therefore, although large aneurysms with a maximum diameter of $\geq 10-12 \mathrm{~mm}$ may be occluded by coil embolization, the risk of recurrence is high and T- or Y-stenting should be considered. For large aneurysms, it is also important to consider the strategy of placing a stent expected to have flowdiverting effects, such as an LVIS blue, or a stent that has properties to straighten the vessel and move the inflow zone such as the Enterprise 2 (Johnson \& Johnson, Raynham, Miami, FL, USA). Half T-stenting is selected for aneurysms with a maximum diameter of $\leq 9 \mathrm{~mm}$ when it is 

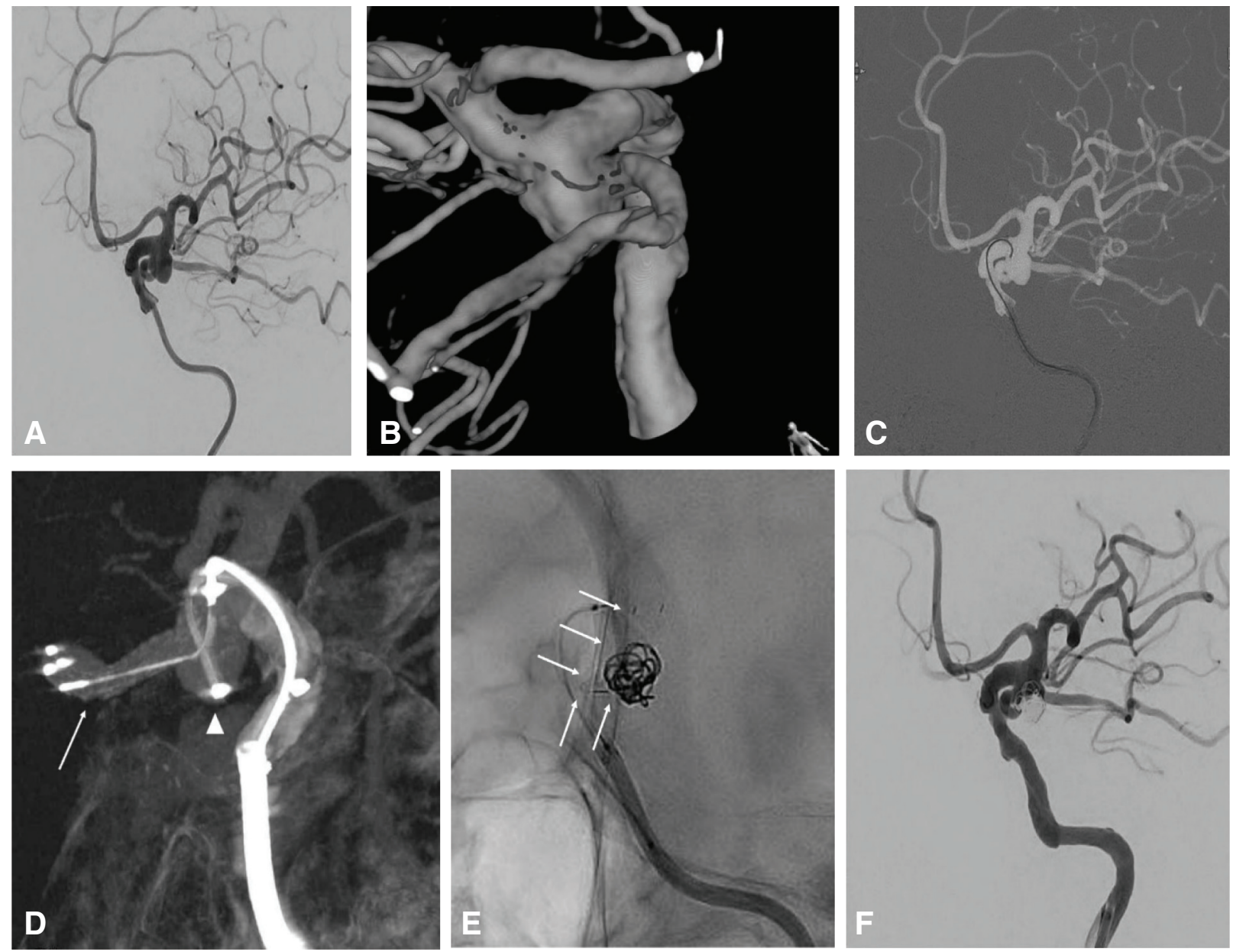

Fig. 3 (A, B) DSA (working angle) and reconstructed 3D-RA of the right ICA. The unruptured aneurysm was located at the right ICA-Pcom. (C) Images before and after navigation of the pigtail-shaped MC. (D) Cone-beam CT after stent deployment (arrow: P-com, Neuroform Atlas $4.0 \times 21$ ) and $M C$ navigation (arrowhead). (E) Images after introduction of the first coil followed by stent deployment (arrow: P-com, Neuroform Atlas $4.0 \times 21)$. (F) DSA just after embolization. The aneurysm was obliterated with a NR. 3D-RA: 3D-rotational angiography; ICA-P-com: internal carotid artery-posterior communicating artery; MC: microcatheter; NR: neck remnant judged that the branching vessel and the neck can be preserved by stenting, and that coil embolization can be performed safely without using other adjuvant techniques.

The use of multiple stents was reported to increase thromboembolic complications, ${ }^{8-10)}$ but it may cause less thrombotic complications than the X- or Y-stent technique because of less overlapping of stents. ${ }^{5,13)}$ However, the angle of bifurcation is often sharp, and considerable skill is required in stent deployment to guide a microcatheter (MC) and adjust the position of the stent tip. In particular, if a branching vessel arises from the dome, as in the present case, securing the vessel is difficult. However, securing the periphery becomes possible by estimating the shape of the $\mathrm{MC}$ by $3 \mathrm{D}$ imaging and shaping the stent into a pigtail or $\mathrm{S}$ shape. Adjustment of the position of the distal end is highly important in stenting, but as the vessel is stretched after guiding of the $\mathrm{MC}$, accurate determination of the position of stent deployment becomes difficult. Therefore, it is necessary to measure the distance from the branch that serves as a marker by digital subtraction angiography (DSA) before guiding the $\mathrm{MC}$ and determine the position of deployment of the distal end. In stent deployment, irrespective of the stent type, we use 21-mm-long stents to prevent slipping if the vessel is markedly tortuous. We use the NF in all patients for stenting of bifurcated vessels. The $\mathrm{NF}$ is disadvantageous in that it cannot be resheathed, but it can be deployed by a simple procedure consistently even in curved parts, has an excellent affixation to vessels, and exhibits minimal shrinking. Therefore, adjustment of the position of its proximal end is not difficult unless it is initially deployed at the wrong site. Although resheathable and easily deployable stents, such as the LEO Baby (Balt, Montmorency, France), are available overseas, if the Enterprise 2 is used among the stents available in Japan, a large 
$\mathrm{MC}$ is necessary, which may make securing the branching vessel and guiding the catheter difficult. Moreover, if an LVIS Jr. (Microvention TERUMO) is used, skillful MC manipulation is necessary for stent deployment in curved parts of the vessel and failure in deployment may lead to vascular occlusion. The minimum diameter of branching vessels that can be stented is $1.5 \mathrm{~mm}$ based on our experience and stenting of thinner vessels is considered to be more dangerous. In addition, in deployment of the proximal end of the stent (distal neck of the aneurysm), the stent is deployed distally into the parent artery. Therefore, a technique to deploy the stent by pushing the $\mathrm{MC}$ in a looped manner distally into the parent artery (MC looping technique $)^{14)}$ or to prevent sliding of the stent into the aneurysm, implementation of partial or complete intra-aneurysmal embolization before complete deployment of the stent is necessary.

As this technique is applied to wide-necked large aneurysms, there is a risk of recurrence, as in Case 1; therefore, it is necessary to evaluate treatment options in the event of recurrence. In consideration of the risk of thrombotic complications, the use of a single stent is preferable. However, in cases in which the neck cannot be covered sufficiently by the half T-stent technique, such as in Cases 1 and 2, T-stenting is inevitable. In principle, it is considered better to place a braided stent, which is expected to have flow-diverting effects, in the parent artery. Although there are indications for treatment using a flow diverter (FD), complete occlusion is difficult to achieve in aneurysms with a fetal-type P-com, ${ }^{15)}$ and if an aneurysm has a branching vessel that cannot be occluded, the placement of the FD should be carefully evaluated. In Case 1, a braided stent was unable to be placed in the initial treatment because of marked kinking of the vessel at the neck of the aneurysm. We considered the possibility of recurrence at the time of initial treatment and planned to place an LVIS blue as a stent-in-stent by the scaffolding technique $^{16)}$ in the event of recurrence. After retreatment using the LVIS blue, no recurrence was noted.

As these techniques are targeted to wide-necked large aneurysms, long-term follow-up is considered necessary.

\section{Conclusion}

For wide-necked ICA-P-com aneurysms with a fetal-type inoccludable P-com arising from the aneurysm, embolization using the T- or half T-stent technique is considered an alternative endovascular treatment method.

\section{Disclosure Statement}

The authors declare no conflicts of interest.

\section{References}

1) Chen Z, Niu Y, Tang J, et al: Endovascular treatment of posterior communicating artery aneurysms in the presence of the fetal variant of posterior cerebral artery. Interv Neuroradiol 2015; 21: 456-461.

2) Cho YD, Lee WJ, Kim KM, et al: Stent-assisted coil embolization of posterior communicating artery aneurysms. AJNR Am J Neuroradiol 2013; 34: 2171-2176.

3) Cho YD, Kang HS, Kim JE, et al: Modified protection using far proximal portion of self-expandable closed-cell stents for embolization of wide-necked intracranial aneurysms. Neuroradiology 2014; 56: 851-857.

4) Liu YQ, Wang QJ, Zheng T, et al: Single-centre comparison of procedural complications, clinical outcome, and angiographic follow-up between coiling and stent-assisted coiling for posterior communicating artery aneurysms. J Clin Neurosci 2014; 21: 2140-2144.

5) Aydin K, Sencer S, Barburoglu M, et al: Midterm results of T-stent-assisted coiling of wide-necked and complex intracranial bifurcation aneurysms using low-profile stents. J Neurosurg 2017; 127: 1288-1296.

6) Morinaga Y, Nii K, Hiraoka F, et al: Three cases with widenecked cerebral aneurysms in whom the T-stent technique was useful. JNET J Neuroendovasc Ther 2018; 12: 409-415.

7) Yagi R, Miyachi S, Hiramatsu R, et al: A case of large broad-necked aneurysm at the posterior communicating artery treated with T-stent technique. JNET J Neuroendovasc Ther 2017; 11: 272-276.

8) Akgul E, Aksungur E, Balli T, et al: Y-stent-assisted coil embolization of wide-neck intracranial aneurysms. A single center experience. Interv Neuroradiol 2011; 17: 36-48.

9) Cho YD, Park SW, Lee JY, et al: Nonoverlapping Y-configuration stenting technique with dual closed-cell stents in wideneck basilar tip aneurysms. Neurosurgery 2012; 70: 244-249.

10) Kim TG, Kim SH, Cho KG, et al: Endovascular treatment of internal carotid-posterior communicating artery widenecked aneurysm using 2 Enterprise stents in Y-configuration. Clin Neurol Neurosurg 2013; 115: 1117-1120.

11) Cho YD, Kim KM, Lee WJ, et al: Retrograde stenting through the posterior cerebral artery in coil embolization of the posterior communicating artery aneurysm. Neuroradiology 2013; 55: 733-739.

12) Park JW, Cheong JH, Choi KS, et al: Retrograde stent-assisted coil embolization of wide-neck or branchincorporated posterior communicating artery aneurysm. J Cerebrovasc Endovasc Neurosurg 2016; 18: 124-128. 
13) Kono K, Terada T: Hemodynamics of 8 different configurations of stenting for bifurcation aneurysms. AJNR Am J Neuroradiol 2013; 34: 1980-1986.

14) Jeon HJ, Park JH, Lee JY, et al: Endovascular coiling for a wide-neck bifurcated aneurysm with anterograde horizontal stenting via microcatheter looping: a technical case report. J Cerebrovasc Endovasc Neurosurg 2018; 20: 181-186.
15) Rinaldo L, Brinjikji W, Cloft H, et al: Effect of fetal posterior circulation on efficacy of flow diversion for treatment of posterior communicating artery aneurysms: a multi-institutional study. World Neurosurg 2019; 127: e1232-e1236.

16) Ocal O, Peker A, Balci S, et al: Placement of a stent within a flow diverter improves aneurysm occlusion rates. AJNR Am J Neuroradiol 2019; 40: 1932-1938. 